\title{
A Teoria do Self Dialógico em Perspectiva
}

\author{
Sandra Ferraz de Castillo Dourado Freire ${ }^{1}$ \\ Angela Uchoa Branco \\ Universidade de Brasília
}

\begin{abstract}
RESUMO - A Teoria do Self Dialógicovem apresentando alternativas ao estudo da pessoa em processos de mudança por meio de recursos teóricos, conceituais e metodológicos que enfatizam o aspecto relacional e plural do self. No presente artigo, objetivaseapresentar e analisar os fundamentos, aplicações e desdobramentos da teoria no intuito de ampliar a discussão teórica acerca do self em uma perspectiva dialógica e desenvolvimental. Analisam-see discutem-se os desafios conceituais e metodológicos enfrentados atualmente pela expansão da abordagem dialógica de self. Destaca-se a necessidade de conceder mais ênfase aos processos semiótico-afetivos e à perspectiva desenvolvimental, bem comode ampliar os contextos de aplicação com alternativas teórico-metodológicas para além da narrativa, considerando situações de intensa socialização, como família e escola.
\end{abstract}

Palavras-chave: self dialógico, desenvolvimento humano, psicologia cultural

\section{The Dialogical Self Theory in Perspective}

\begin{abstract}
The Dialogical Self Theory, since its publication in the early 1990s, has presented alternatives to the study of personsundergoing psychological changes by offering theoretical, conceptual and methodological resources that emphasize self's relational and plural aspects. This paper aims at presenting and analyzing the theoretical and methodological foundations, applications and possibilities of the theory in order to enrich the discussion about the self towardsa perspective that integrates both, dialogical and developmental dimensions involved in psychological changes. The critique underlines the need for emphasizing semiotic-affective and developmental processes, and offering resources that go beyond narrative analyses that enable the study of human development within diverse settings like family and school.
\end{abstract}

Keywords: dialogical self, human development, cultural psychology

A Teoria do Self Dialógico vem construindo referenciais teórico-metodológicos para estudar as relações entre o eu, o mim e o meu a partir de uma perspectiva, como sua própria denominação indica, dialógica. Neste artigo objetiva-se, de forma ampla, apresentar e analisar os fundamentos, aplicações e desdobramentos da abordagem. Para isso, propõe-se uma sistematização de sua trajetória histórica; a caracterização de seus elementos constituintes; uma reflexão sobre sua metodologia e a discussão sobre os desafios conceituais e metodológicos enfrentados atualmente pela expansão da abordagem dialógica de self. Destaca-se, ainda, a necessidade de conceder mais ênfase ao sistema semiótico e à perspectiva desenvolvimental, além de ampliar os contextos de aplicação por meio do desenvolvimento de alternativas teórico-metodológicas para além da narrativa, desde que se mantenha como foco o princípio dialógico e relacional.

$\mathrm{O}$ termo self refere-se, em português, à pessoa, ao eu, a si próprio. Como categoria psicológica, é utilizado no sentido de identidade, personalidade, ego. É também considerado uma categoria reflexiva referente a si mesmo: o prefixo auto em: autoestima, autoconceito, autoconfiança, autoimagem e autodeterminação. Indica a unidade da psique humana que permite que o eu possa falar de mim, conduzir minha ação e relacionar-se com o que é meu. É o que permite à pessoa situar-se em diferentes contextos vividos e nas interações interpessoais. A qualidade da relação entre o eu, o mim e o

1 Endereço para correspondência: SQS 402, Bloco H, Apto. 304, Asa Sul, Brasília, DF, Brasil. CEP: 70.236-080.E-mail: sandra.ferraz@ gmail.com meu é o foco dos estudos de self. Entretanto, consideramos que "o que determina a fronteira entre um self e um nãoself é a atitude emotiva em relação a um objeto ou ideia" (Barresi, 2002, p. 239) que emerge e se desenvolve na relação com o outro. Isso significa que o eu, o mim e o meu devem ser contextualizados nas relações e nas experiências sócio-afetivas.

Por isso, a função comunicativa da linguagem é tão importante, como componente essencial para a constituição do self e como recurso para a análise e a interpretação dos processos psíquicos. Por meio das interações comunicativas,em especial, na narrativa, as relações entre o eu, o mim e o meu se materializam em forma de posicionamentos. A partir do significado afetivo das experiências ao longo da vida desde a infância e das posições sociais assumidas ao longo do tempo, os posicionamentos participam de forma relativamente estável das dinâmicas das relações da pessoa com o seu meio e consigo mesma.

Nesse sentido, as formas narrativas, que são representativas do significado socioafetivo dos diferentes posicionamentos, também passam a desenvolver mecanismos de autorregulação.Em nossa concepção, esses mecanismos, com seus diversos níveis de complexidade, são embevecidos na cultura e são elaborados nas narrativas e autonarrativas,nas quais a qualidade da relação entre os três componentes do self - o eu, o mim e o meu-é mais visível, porém, não a única como argumentaremos adiante. Optamos por utilizar o vocábulo em inglês visando à consistência com a literatura e, também, diante do fato de que o uso do plural dos termos 
eu e mim soa particularmente estranho na língua portuguesa, sem querer, com isso, sugerir um colonialismo linguístico. Além do mais, o self tem experimentado um desenvolvimento teórico que dificulta uma tradução sem que haja uma dissociação do seu lócus conceitual.

\section{A Teoria do Self Dialógico e seus Fundamentos: James, Mead e Bakhtin}

William James (1842-1910) situava o self como um composto bidimensional formado por eu(I-Self)e mim (Me-self)(James, 1890/2007). O eu se refere à pessoa como sujeito, o eu-conhecedor. $\mathrm{O}$ mim se refere à pessoa como objeto, o eu-conhecido. Como um dos fundadores do pragmatismo e adepto ao empiricismo, James defendia que todo o estudo psicológico deveria ser abordado a partir de sua consequência ou efeito concreto. Denominou o mim a parte empírica do self, relacionando-a ao mundo de objetos e pessoas vinculadas ao sujeito concretamente; seria a extensão de si mesmo refletido no contexto socioambiental. James identificou a manifestação de vários selves, classificando-os em self material, self social e self espiritual (Barresi, 2002; James, 1890/2007). A relação entre o eu e o mim dar-se-ia em um contínuo, de forma fluida e dinâmica: enquanto o eu conduz as ações e oferece interpretações, o mim representa a dimensão reflexiva do sujeito que realiza as ações específicas segundo o script oferecido. Em consonância com a dialética histórica e contínua de Heráclito de que uma ação gera uma consequência e, portanto, outra ação em um fluxo contínuo, a visão de self de James não contempla opostos nem descontinuidades, embora reconheça a dimensão essencialmente social e concreta do composto eu-mim.

George Herbert Mead (1863-1931), conterrâneo e contemporâneo de James, incorporou ao estudo do self as noções de atores sociais, de significado e de símbolo (Mead, 1913, 1934/1967). Ambos fizeram parte do primeiro período da Escola de Chicago, à qual também pertenceu John Dewey (1859-1952).Meadera adepto à visão dialética hegeliana e considerava todo o ato humano um ato social voltado para a perspectiva do outro na experiência. Destacou a função dos processos sociais de comunicação e, por conseguinte, do significado. Esses seriam responsáveis por permitir o desenvolvimento do self a partir da experiência. Elaborou a noção do eu $(I)$ como autor e narrador e o conceito de mim $(\mathrm{Me})$ como o ator, aquele que desempenha papéis específicos em suas interações com os demais. Assim, o eu (I-Self) é o sujeito, o autor que pode imaginar o futuro e reconstruir o passado, pois é ele quem provê uma compreensão do mim $(\mathrm{Me}$-Self). A noção de ator-personagem define a característica reflexiva do self (Hermans, 2001a), em que linguagem e consciência conferem ao sujeito a capacidade de se ver como objeto na experiência (Mead, 1934/1967). Dessa forma, o self se relaciona com a constituição de si na ordem do sujeito.

A metáfora literária de $\operatorname{Mead}(1913,1934 / 1967)$, que utiliza a noção de personagem para explicar a constituição dinâmica do self, tem consonância com os estudos de Mikhailovich Bakhtin (1895-1975). Suas ideias, fundamentadas na filosofia marxiana, concebiam a língua como signo social e ideológico e o desenvolvimento da consciência a partir da interação social (Bakhtin, 1992). Desenvolveu as noções de vozes e de romance polifônico, que foram logo incorporadas à fundamentação da Teoria do Self Dialógico junto a outrosconceitos bakhtinianos, como ambivalência, bidirecionalidade e dialogismo(Hermans, 1996a; Hermans \& Kempen, 1993; Hermans, Rijks, \& Kempen, 1993). No contexto literário, Bakhtin lançou as bases para a definição de dialogismo ao contemplar a interpretação participativa do leitor na compreensão da obra (Barresi, 2002; Holquist, 1990).

A partir das posições de $I$-Self de James, das contribuições de Mead sobre o self autor-ator de uma história e, principalmente, das noções de pluralidade de discursos (polifonia) e de posições de self desenvolvidas por Bakhtin, Hubert J. Hermans mobilizou e organizou os fundamentos do que denominou Self Dialógico (Hermans \& Dimaggio, 2004).No caso da Teoria do Self Dialógico, o conceito de dialogismo é utilizado, no contexto clínico, no sentido de (a) reconhecer a participação do cliente e do psicólogo nos processos de interpretação, tendo em vista o desenvolvimento do self (Hermans, 1987); e (b) de conceber a emergência e o desenvolvimento dos diferentes posicionamentos do self sob uma perspectiva dialógica no processo de interação (Hermans, 2001b; Hermans \& Kempen, 1993).

Assim, a Teoria do Self Dialógico emergiu na década de 1990 e consolidou a abordagem do self em uma perspectiva relacional, dialógica e dinâmica.A entrada no circuito de produções internacionais e a diversidade de trabalhos na linha do self dialógico, no entanto, diversificaram e propuseram desafios teóricos, bem como questões epistemológicas e metodológicas a partir dos anos 2000. Muitos destacaram as múltiplas perspectivas culturais que desencadearam reformulações e ampliações, como os estudos deSunil Bhatia (Bhatia \& Ram, 2001), Shui Fiona Chan (Chan, Ho, Peng,\& Ng, 2001), Nandita Chaudhary (2003; Chaudhary \& Sriram, 2001), Miguel Gonçalves e João Salgado (2001; Salgado, 2006)e Jaan Valsiner $(2001,2007)$, dentre outros(Hermans\& Damaggio, 2007).

\section{Aspectos Teórico-Conceituais}

A relação I-self do self dialógico é, como vimos, relacional e situacional. Quando o I (em termos de ideia, pensamento, significado) é contextualizado em um tempo e espaço específicos, então temos a materialização de um self por meio de uma posição corporificada do I (embodied self) (Hermans, 1992). Por exemplo, na escola, a criança assume uma posição de estudante. Na negociação concreta do sujeito com o contexto, constituem-se os vários selves, que se estabelecem na e pela interação. Cada self, portanto, está constituído de um $I$ (que assume a qualidade de agente) e dos vários selves que venham a emergir e a desenvolverse mediante o estabelecimento de relações significativas com o outro (ou outros) no contexto da experiência. As possibilidades de relação são oferecidas pelas situações concretas vivenciadas pela pessoa. Contextos culturais específicos, como escola, família e trabalho, caracterizam-se por um conjunto de práticas socioculturais que disponibilizam lugares que já trazem definidas, em linhas gerais, as posições 
dos sujeitos. Porém, a Teoria do Self Dialógico se interessa pela forma singular e característica como cada sujeito assume essa posição. Na medida em que o mim, que é responsável por fazer a transição para o meu, provê o I com informações necessárias para a interpretação e ressignificação de si, do objeto e da experiência, há uma relação da ordem do significado que se constitui simultaneamente entre o I e os vários selves, de forma bidirecional, gerando a realidade própria e característica do self (Hermans, 1996b).

Eminentemente social, a existência do self implica a existência de alguém significativo, ou um não-self (Barresi, 2002), em função de quem o self, após um determinado período de experiência, relaciona-se com uma configuração de I-Selves relativamente estruturada, porém, dinâmica (Hermans, 1992, 1996b; Hermans \& Hermans-Jansen, 1995). É importante destacar o caráter social do self dialógico. O social não se refere à interação social concreta, mas à possibilidade de definir perspectivas alternativas de posicionamento em função de um "outro social", real ou fictício. Acrescentaríamos o outro social que estaria tanto fora de mim como dentro de mim no caso do estabelecimento de diálogos entre diferentes posições do eu (I-Positions).

A noção de voz está relacionada ao pressuposto de que há uma relação entre pensamento, sentimento e ação que, ao manifestar-se na experiência, constitui uma materialização do self. A voz refere-se a uma unidade de significado, um símbolo, um ponto de vista, uma posição pessoal-Iposition - ou um personagem, um self-position específico (Bakhtin, 1992; Hermans, 1996b). Na narrativa de uma pessoa, cada voz pode ser identificada por meio das palavras ditas - sobre tópicos, atitudes, objetos, relacionamentosmas é na forma em que são expressas que podemos identificar significados, sentimentos e emoções, que, por sua vez, informam sobre a experiência ou conjunto de experiências do self. Dessa forma, a posição institucional de estudante, por exemplo, difere substancialmente das posições experimentadas por cada estudante particular. Face às diferentes experiências escolares, o self desenvolve um conjunto de formas de se relacionar baseadas em diversas vozes de acordo com os diferentes selves experimentados.

$\mathrm{Na}$ Teoria do Self Dialógico, há dois tipos de selves: um representa o romance composto por várias vozesrepresentado pelo sujeito singular, que se constitui e se expressa de forma dialógica-e o outro representa a voz. O self materializado, empírico, expresso por uma voz, é a extensão de uma posição do eu (I-position). Ao mesmo tempo, várias I-positions formam um sistema de valoração que é o próprio self (Hermans \& Kempen, 1993; Hermans, Kempen,\& Van Loon 1992; Hermans et al., 1993). Para Hermans, as vozes, que estão relacionadas com I-Positions típicas em determinada situação, apresentam-se de forma descentralizada e múltipla, criando e se movimentando por campos dinâmicos de significados. As diversas negociações, contradições e integrações do self como I-sistemaromance-autor-resultam em e articulam uma variedade de significados ou vozes (Hermans 2001a, 2001b). Tal multiplicidade inclui dimensões diversas relacionadas a todas as coisas como pessoas, objetos e experiências significativas expressas pela pessoa na narrativa (Chaudhary, 2003). Em outras palavras, tudo referente ao eu, ao mim, ao meu fazem parte do self e dialogam entre si constantemente sem que isso signifique coerência, consistência e coesão. A Teoria do Self Dialógico se preocupa especificamente em identificar as I-Positions e suas relações dialógicas, promovendo, assim, o desenvolvimento de um self como romance melhor conhecido, mais organizado e coerente com o sujeito- 0 cliente em contexto clínico-, o que requer níveis de intencionalidade por parte do sujeito.

A noção de polifonia expressa duas idéias: a ideia de que um self consiste de diferentes vozes ou Self-Positions — significados, posicionamentos, pontos de vista — assim como a ideia de que um self possui muitas posições do eu (I-Positions) - eu como profissional, como estudante, como filho, como cônjuge, como representante político,entre outros. Isso ocorre devido à multiplicidade de experiências, situações e realidades vividas e construídas (Hermans, 1996b; Hermans \& Kempen, 1993; Hermans et al., 1992) pelos sujeitos em interação, em contexto socioculturais específicos. Mas, além da composição múltipla do self, Hermans (1996b) destaca a concepção essencialmente dialógica de polifonia desenvolvida por Bakhtin, para quem as vozes (personagens) são plurais, contraditórias, conflitantes e mesmo opostas (Bakhtin, 1992; Barresi, 2002; Holquist, 1990), apesar de relativamente organizadas em um sistema dinâmico. Em publicação posterior, Hermans (2001a) explica que a 'polifonização' precede a constituição do self. MarieCécileBertau (2004) esclarece que:

Antes que as vozes se tornem I-Positions, existe a voz concreta e perceptivel do outro que, primeiramente, forneceu ao self emergente uma estrutura significativa. Esta voz (ou vozes) envolve o self em desenvolvimento na dialogicidade, devido a sua ligação com uma estrutura do tipo fala-resposta, e com a outra pessoa. (pp. 39-40)

Uma vez constituído, o self é um sistema organizador das múltiplas vozes (Hermans, 2001a). A narrativa e a comunicação intersubjetiva são essenciais para a concepção dialógica das múltiplas vozes, especialmente por conceber e identificar as relações de oposição e dominação entre as vozes. Os construtos pessoais são articulados na construção e movimentação dos personagens ou vozes. A movimentação desses personagens e a caracterização dos cenários podem obedecer à contextualização espacial (justaposição de episódios) ou a uma contextualização ao longo do tempo (Hermans, 1996a). Além disso, é fundamental conceber o diálogo e as negociações que se estabelecem entre as diferentes vozes (referente às I-Positions), no sentido de configurar o desenvolvimento e a dinâmica característicos do self dialógico, como veremos a seguir.

A noção de self dialógico implica intercâmbio entre vozes mutuamente influentes (Hermans, 1996b). Em oposição ao self estático, mecanicista e centralizado, a perspectiva dialógica permite transcender a visão individualista e racionalista (Hermans et al., 1992) e a dicotomia individualismo-coletivismo (Chaudhary \& Sriram, 2001) ao integrar processos inter e intra-psicológicos em experiências concretas ou imaginárias (Valsiner, 2001). Nesse intercâmbio, caracterizado por processos de negociação, observa-se que as vozes são dinâmicas e costumam alterar suas posições no decorrer das experiências concretas, o que é muito importante para o processo de organização do self. Assim, 
explica-se a noção de polifonização. Na medida em que o self experimenta as diferentes posições, até mesmo opostas de seus vários selves, ele vai se organizando e definindo-se progressivamente. Há, portanto, que se considerar que o self é essencialmente descentralizado na narrativa e, quando há a omissão do narrador, é mais provável que exista um conflito interno entre as diferentes vozes (Hermans \& Kempen, 1993). Entretanto, o self deve ser capaz de lidar e integrar, em certa medida, as diferentes vozes.

Nandita Chaudhary e Sujata Sriram (2001) sintetizam esse processo, explicando que a multiplicidade de vozes que dá forma ao self inclui histórias que articulam passado, presente e futuro na diversidade de papéis sociais (I-Positions) que aparecem em conjunto e em interações. Pressupondo que diferentes posições pessoais, por sua vez, indicam diferentes sistemas de valores, consideram que o sistema de self, embora dinâmico, tem a função de integrar e processar: (a) significado ou valoração, (b) conotação afetiva correspondente em suas formas latente e manifesta, (c) padrão de afetos e (d) nova valoração ad infinitum. Quando algumas posições, especialmente as consideradas 'más' ou 'inadequadas' socialmente não são bem organizados nos sistema de self, têm-se uma desordem que impede o funcionamento saudável do self (Hermans \& Dimaggio, 2004). Compreender o self em sua dialogicidade é, pois, fundamental para explicar sua natureza plural, e muitas vezes contraditória, em contínuo processo de desenvolvimento.

\section{Aspectos Metodológicos}

A Teoria do Self Dialógico passou a abordar o self como um sistema semiótico organizado, expresso e reorganizado na narrativa, consolidando seu método de autoanálise das diferentes posições de self (Self-Positions) assumidas pela pessoa e em intenso diálogo com o psicoterapeuta. $\mathrm{O}$ conceito de valoração refere-se ao processo pelo qual a pessoa atribui significado às próprias experiências e, para isso ser possível, o cliente toma parte ativa no processo de investigação do self (self-investigation) por meio da autoconfrontação (Hermans, 1989, 2002; Hermans \& Damaggio, 2007).

A valoração [valuation] refere-se a qualquer coisa que a pessoa julgue importante para a sua vida diária. O método da autoconfrontação é utilizado como uma forma de tornar explícito o sistema de valoracão da pessoa, com especial atenção às suas propriedades afetivas. O método mostra como o sistema é organizado e reorganizado através do tempo. (Hermans, 1987, p.10)

O processo de valoração se tornou, por assim dizer, uma forma de acessar aspectos significativos do self nas experiências cotidianas. O conceito de valor pode ser considerado positivo, negativo ou ambivalente devido à a dualidade básica da natureza humana. O sistema de valoração implica a existência de diferentes valores na perspectiva de uma posição pessoal. Assim, é possível identificar os motivos básicos (basic motives) que levam o self a articular determinadas posições. Os motivos básicos não são autoexplicações, mas temas que aparecem nas autonarrativas indicadores de posições positivas ( $S$ motives) ou negativas (O motives) no posicionamento do self perante um obstáculo. Um motivo básico positivo indica maior centralização no self- autoafirmação, automanutenção, autoprojeção etc. - - enquanto o motivo básico negativo está direcionado ao outro-à realização de um não-self. Entretanto, o que define a funcionalidade do motivo básico é o resultado da ordem do sentimento que cumpre a função de retroalimentar aquele motivo. Para Hermans (2001a), essa relação se dá da seguinte maneira: motivo básico $\rightarrow$ desejo $\rightarrow$ obstáculo $\rightarrow$ sentimentos (positivo: superação; negativo: frustração) $\rightarrow$ motivo básico.

Quando a valoração representa uma realização do $S$ motive (Eu passei num teste muito difícil), a pessoa experimenta um sentimento de força e orgulho em conexão com a valoração. De forma similar, quando uma valoração representa a realização de um $O$ motive (Eu gosto quando meu filho toca piano), sentimentos de intimidade e ternura são associados com a valoração. Em outras palavras, a base motivacional latente torna-se manifesta no padrão afetivo associado com a valoração particular. (Hermans, 2001a, p. 348)

É necessário observar que o qualificativo positivo ou negativo do motivo não significa a qualificação da relação, do motivo ou sentimento em bom ou mau para o self, mas indica se o motivo que mobiliza a posição do 'eu' está direcionado ao centro do self ou ao outro.

A organização e reorganização do self ao longo do tempo é o que Hermans (2001a, 2001b) denominou de repertórios de posições pessoais (RPP) e suas diversas conotações afetivas. A partir da narrativa, os repertórios se definem por padrões identificados nas mudanças e na dinâmica das posições pessoais e seus significados/valores correspondentes. Porém, há um grande número de posições no interior e na superfície do repertório que devem ser investigadas. Para isso, o autor enfatiza os seguintes aspectos na narrativa: (a) relacionamento entre autor-ator/protagonista; (b) perspectiva cronológica (contexto temporal); e (c) justaposição de histórias (contexto espacial).

A noção de campos é importante para compreender a relação entre posições externa e interna do self, segundo Hermans (2001a), e que irá viabilizar o desenvolvimento de uma metodologia de pesquisa. Ele propõe um campo composto por dois outros, representados por círculos, um dentro do outro. O círculo de dentro representa a parte interna do self e o de fora, a parte externa. Há também a parte de fora do campo, onde se situam os aspectos não percebidos pelo self. As duas dimensões representadas no campo referemse aos aspectos internos (mim) e externos (meu) do self, assim como indicam as relações entre o self e o mundo. $\mathrm{O}$ encontro entre o interno e o externo se dá no processo de negociação, cooperação, oposição, conflito, acordos e desacordos, constituindo os diferentes selves do sistema. Assim, é possível relacionar um conjunto de referências do self interno e externo a uma I-Position específica.

A partir da relação entre posições internas e externas contidas na narrativa da pessoa, constrói-se o RPP. Os padrões de posicionamento se baseiam em diferentes conceitos referentes a si mesmo (o self interno) e nas relações que o self estabelece com os outros significativos (o self externo). O psicoterapeuta elabora um quadro com uma lista de conceitos referente ao self interno e uma lista de pessoas referentes ao self externo. Por meio delas, tem início o método de autoconfrontação: primeiramente o cliente se defronta 
com o quadro contendo as listas de RPP e atribui valores de relevância às relações entre os conceitos das duas listas de acordo com uma escala predefinida. Com base nessas escalas, um segundo quadro é gerado, desta vez com uma só lista, com a relação entre as posições internas e externas sinalizadas como as mais relevantes. A autoconfrontação utiliza-se do recurso do RPP para intermediar o diálogo entre o cliente e o psicoterapeuta. Segundo Hermans (2001a), a contextualização do processo de posições pessoais internas e externas permite as seguintes relações: (a) o grau de proeminência (relevância) de uma posição interna é obtido pela quantidade de posições externas que ela mobiliza; e (b) uma posição externa é relevante na medida em que permite acessar um maior número de posições internas daquele repertório. Proeminência se refere à dinâmica e ao caráter relacional das posições internas e externas. Além disso, afirma que é possível identificar e relacionar posições correspondentes e posições de oposição.

A metodologia possibilitou constatar que (a) "quanto mais importante é uma pessoa significativa para alguém, mais posições internas são evocadas no contato com esta pessoa" (Hermans, 2001a, p. 329); (b) as relações de oposição permitem a construção de significados: uma posição é significativa quando o seu contraponto está assinalado, ou seja, há uma posição oposta; (c) é necessário estabelecer uma relação entre determinadas posições pessoais e posições sociais, em termos de papel, demandas e expectativas sociais, pois, em muitas sociedades ou grupos, algumas posições pessoais internas são mais valorizadas do que outras; (d) no caso de posições pessoais de baixa proeminência, há muitos significados: ou ela não é relevante para o RPP, ou ela é internamente muito importante mas não é evocada pelos outros (pessoas significativas), ou então ela é uma posição suprimida ou escondida; e finalmente (e) uma posição pode ser empurrada para o background, mas ainda ser influente por meio de outras posições e significações. Aqui é o caso de analisar em qual (ou quais) posição (ões) poderíamos situar a dimensão, por exemplo, dos preconceitos. Hermans conclui que a metodologia do RPP atende o caráter espacial do Self Dialógico, em que "o espaço é básico para a voz e o diálogo, e mesmo precede estas capacidades (capacities), tanto de uma perspectiva ontogenética como filogenética" (Hermans, 2001a, p. 333).

A teoria também prevê uma matriz hierárquica para o estudo das posições internas e externas baseadas nos significados e valores, assim como na conotação afetiva de cada posição. Significados e valores são equivalentes e vistos de forma processual. A ideia é que uma posição tem múltiplos significados e conotações afetivas, sendo essas indissociáveis das situações vividas pela pessoa. A identificação das conotações afetivas é importante porque essas podem ser vistas como a expressão explícita de um motivo de fundo psicológico. Daí a importância da narrativa na identificação dos repertórios de posições pessoais e das diversas conotações afetivas.

Os participantes ... são considerados experts no significado que eles atribuem aos eventos de sua própria vida, e bem como conhecedores das circunstâncias particulares e eventos que desempenham um papel mais importante em sua história pessoal... Enquanto a formulação das valorações se fundamenta primariamente na expertise dos participantes, a exploração dos significados afetivos das valorações é mais um produto da expertise metodológica do psicólogo. (Hermans, 2001a, pp. 342-343)

De forma sucinta, nos tópicos anteriores, buscamos trazer as principais características de um processo de quase três décadas de desenvolvimento de uma metodologia que consolidou a perspectiva dialógica do self. O debate atual do fenômeno dialógico do desenvolvimento humano pela comunidade científica internacional tem demonstrado a utilidade conceitual da Teoria do Self Dialógico no contexto de várias orientações psicológicas (Hermans \& Dimaggio, 2004). Entretanto, a expansão da teoria para outros contextos culturais e campos científicos requerem um redimensionamento quanto algumas questões epistemológicas e metodológicas. $\mathrm{Na}$ perspectiva do desenvolvimento humano e da Psicologia Cultural, discutimos três dimensões: o fundamento pragmático; o confronto da ideia de selfe suas metodologias com diferentes sistemas culturais e problemas da relação I-Self.

\section{Desafios Conceituais, Metodológicos e Epistemológicos}

O foco de uma teoria desenvolvida na prática clínica e voltada somente para a perspectiva da experiência pessoal apresenta alguns desafios da ordem da compreensão do próprio sujeito, o qual passa a ser reconhecido "apenas na e através da sua participação em tais práticas" (Colapietro, 2006, p. 26). Além do mais, “a face pragmática do self dialógico é apenas este participante responsivo em uma prática humana, especialmente em um momento crítico, i.e., um momento de crise" (Colapietro, 2006, p. 25). A interpretação de um self gerado nesse contexto exige uma postura crítica face às informações construídas em colaboração entre cliente e psicoterapeuta, que têm, no primeiro, o único informante. A narrativa, mesmo sendo poderosa expressão das contradições internas e reveladoras das realidades sociossubjetivas, não chega a cobrir aspectos da experiência que estão fora do alcance da percepção do sujeito. Além do mais, há que se levar em conta a imaginação e a fantasia envolvidas na reflexividade. A contextualização das posições pessoais na qual insiste Hermans (2001a) tem sempre em foco aspectos referentes a um contexto pragmático e funcional.

Primeiramente, nossa crítica ao pragmatismo em que se insere a Teoria do Self Dialógico refere-se à colocação do sujeito em uma função de ator social que atenda à moral dominante, cujo objetivo parece ser o bom funcionamento do indivíduo na sociedade. A teoria parece endereçar uma sociedade em que há uma relativa estabilidade de papéis sociais e padrões de comportamento, sem necessariamente contemplar uma visão epistêmica de ser humano. Se o objetivo do tratamento psicoterapêutico é a mudança no funcionamento daquele self nas relações com o seu meio imediato, cabe mencionar a lacuna existente quanto à dimensão da interdependência sociocultural inerente à visão de um ser humano construtivo e criativo, capaz de transformar não apenas a si mesmo como ao contexto no qual está inserido (Branco et al, 2004; Colapietro, 2006). 
Autores hindus (Chaudhary,2003; Chan et al., 2001) consideram que uma dimensão dialógica e relacional do selfé imprescindível para superar o vácuo entre a psicologia no ocidente e no oriente. Entretanto, destacam os desafios postos pela ideologia de sua cultura, segundo a qual self e desenvolvimento espiritual são, em princípio, antagônicos. Nesse caso, algumas práticas sociais simplesmente não são compatíveis com os exercícios de autorreflexão e autoconfrontação demandados pela Teoria do Self Dialógico.Tal fato coloca em evidência que a elaboração e a produtividade científica de uma teoria e uma metodologia dependem da cultura.

Toda cultura tem coisas significativas a dizer sobre o desenvolvimento do self, bem como sobre o movimento do self em direção a determinados caminhos valorizados pela comunidade. Ao fazer isto, as sociedades provêm canais de expressão para, ou a restrição, relativa à manifestação de uma série de comportamentos, particularmente se estes são impregnados de significação social. A experiência social não é limitada ao condicionamento ou modelação, simplesmente; existe o poder, a persuasão, o diálogo e uma vasta quantidade de outras formas de socialização da criança no sentido de ser ou tornar-se, que teriam impacto sobre os processos de self. (Chaudhary, 2003, p. 475)

Não ter familiaridade com a prática autorreflexiva em termos abstratos não significa que a pessoa não tenha um sentido do self. Muitas vezes, a tarefa de observar e falar sobre si é um ato completamente novo ou, então, mal visto pela cultura de origem. Ao mesmo tempo, devemos considerar as relações de poder, hierarquia, autoridade e ideologia ao lidar com a diversidade cultural e as desigualdades de cada cultura como processos singulares de práticas e de domínio entre centro e periferia ou mesmo entre grupos.

Nossa segunda crítica se dirige à metodologia da Teoria do Self Dialógicocom pessoas pertencentes a grupos culturais com forte ênfase em uma diversidade de elementos externos significativos. A definição de aspectos internos e externos do self-proposta na construção do RPP e da matriz de valoração-pode tornar-se pouco viável em sociedades com sistemas muito complexos de crenças e valores culturais.

A existência de zonas de contato, referentes às zonas geograficamente periféricas da cultura (fronteira, bordas etc.), demanda uma perspectiva que considere a integração entre self e cultura. O mesmo se aplica aos casos de migrações (Bhatia \& Ram, 2001; Chaudhary \& Sriram, 2001). A pessoa que está em zona de transição ou de migração, especialmente aquela que deixa o seu núcleo relacional de origem, deve receber um olhar diferente sob o ponto de vista metodológico do self dialógico. Os motivos, condições e perspectivas da migração devem ser levados em conta, assim como as tendências existentes no grupo cultural de origem (Chan et al., 2001; Chaudhary, 2003).

Os desafios de ordem da cultura apresentados acima são apenas em parte equivalentes aos desafios que um estudo dialógico enfrenta em sociedades altamente diversificadas, que possuem uma miríade de zonas de contato, físicas e simbólicas - em termos de formação étnica, cultural, distribuição de riqueza, economia (industrialização e cultura agrária), escolarização, nível de institucionalização, profissionalização etc. Reconhecemos que é necessário estudar não só uma comunidade ou sociedade, mas todo o contexto de pesquisa com uma 'psicologia pró-cultural' que contemple as dinâmicas entre o centro e suas fronteiras, especialmente, as fronteiras simbólicas (Valsiner, 2001, 2007).

É exatamente nas zonas de contato que se constituem de forma mais intensa as marcações intergrupais, sejam de caráter nacional, étnico, religioso ou de outra natureza. Em sociedades plurais, portanto, com tendências históricoculturais integracionistas (como é o caso do Brasil), as marcações de desigualdades definidas por fatores sociais, econômicos, raciais são estabelecidas e transformadas dentro dos próprios grupos. Daí o grande desafio de abordar um self que enfrenta as dificuldades típicas das relações entre 'zonas de contato’ dentro do próprio grupo de convivência.

Barresi (2002) critica a Teoria do Self Dialógico no que se refere à consistência epistemológica com o dialogismo de Bakhtin e, em decorrência disso, à ideia de flutuação de I-position entre os diferentes personagens ou vozes. O processo de constituição do selfdialógico dá-se exatamente pela relação entre as duas partes: o autor $(I)$ e o ator (self). Isso ocorre mediante um distanciamento relativo e na medida em que a parte do self que executa a ação (a parte que será objeto de reflexão) e a parte que a comanda (atua como agente) entram em interação e se conhecem. No que diz respeito aos atores (selves), esses estão sempre em diálogo, em negociação, e não conseguem, por si só, integrar, convergir, unificar as informações em um só self. De acordo com o princípio polifônico, um $\mathrm{Me}$-self (ator ou personagem ou voz) não pode integrar informações do outro, só pode dialogar com ele, pode dominá-lo ou ser dominado, mas não se fundir com os outros selves.

Se os atores ou personagens não conseguem integrar por si mesmos as informações e valorações dos diferentes selves, o observador ou autor $(I)$ pode fazê-lo. A integração só pode ser feita do ponto de vista do observador $(I)$. Segundo a crítica de Barresi, esse tipo de análise, porém, fica comprometida,porque Hermans designa uma voz ou personagem para cada I-position (autor). O pensamento ou significado é materializado em um $I$, que, ao ser materializado, adquire uma voz, self. Como o I pode flutuar entre os diferentes selves e integrá-los se ele se materializa por uma voz ou self? Na Teoria do Self Dialógico, há, portanto, uma multiplicidade de posições de $I$, para cada qual pode ser dada uma voz, o que permitiria que estas se engajassem em diálogo.

\section{Expansão do Self Dialógico}

Vários trabalhos têm enfatizado o caráter semiótico do sistema de self (self system), integrando os processos de mediação semiótica da perspectiva cultural à identificação e análisedos repertórios de posicionamentos pessoais desenvolvidos pela Teoria do Self Dialógico. Algumas pesquisas (e.g., Duarte \& Gonçalves, 2012; Gonçalves \& Salgado, 2001;Lima de Souza \& Gomes, 2009; Salgado, 2006)destacam funções de ordem simbólica, histórica, material, moral e afetiva para o desenvolvimento da subjetividade em períodos de transitoriedade por meio 
da articulação de várias dimensões do self, integrando os processos de mediação semiótica da psicologia cultural.

Filipa Duarte e Miguel Gonçalves (2012) aliam a interpretação de repertórios de posicionamentos pessoais, utilizando análise do discurso e o estudo da construção dos significadosa partir daanálise microgenética. Primeiramente, selecionam dois repertórios de posicionamento mais proeminente entre os sujeitos que vivem um período de transição ocasionado pela emergência da maternidade. Nos discursos, identificam o embate e o caráter ambivalente entre a posição de mãe e a posição de profissional. Em um segundo momento, analisam o processo da construção de significadopelos sujeitos, enfatizando a elaboração de ferramentas semióticas como forma de lidar com tais ambivalências e as tensões enfrentadas pelos sujeitos em seus momentos históricos individuais antes e depois do nascimento de seus filhos. Em síntese, demonstram a importância de estudar o processo de elaboração de dispositivos semióticos que possibilite ao sujeito dar sentido à complexa e tensa relação entre experiências subjetivas e expectativas culturais. Dessa forma, o sujeito pode lidar com as diferentes posições pessoais, eminentemente sociais e dialógicas, mesmo ou especialmente quando essas rivalizam no sistema semiótico pessoal, ou seja, no sistema de self dialógico.

Apesar de o círculo de pesquisadores que dialoga e que desenvolve pesquisas na perspectiva da Teoria do Self Dialógico ter expandido os referenciais cunhados inicialmente por Hermans e colaboradores, os estudos têm se caracterizado pela análise exclusiva de narrativas pessoais e com um olhar em retrospectiva. Enfim, perguntamonos: será possível incluir alternativas metodológicas para análise dos posicionamentos pessoais que possam ir além das manifestações discursivas reflexivas dos sujeitos e que considerem ações em tempo real desses posicionamentos? Ao mesmo tempo, como desenvolver um modelo que possa representar uma síntese dinâmica dos múltiplos níveis de análise do self dialógico e seus processos de mudança voltados para as possibilidades?

Compreender os processos de construção de significados é crucial para o estudo do self dialógico, pois esse se fundamenta nos processos de significação sem os quais não haverá o desenvolvimento das funções psicológicas genuinamente humanas essenciais para a vida em sociedade. Por isso, aliada à análise do processo de construção de significado interpretado nas narrativas, consideramos que a observação e análise das interações interpessoais em contexto contribuam significativamente para a compreensão do self dialógico como sistema semiótico em um sentido mais amplo, o que passamos a considerar sistema de self dialógico. A partir e em função da convivência com o outro, o sujeito constrói repertórios de posicionamentos pessoais que constituem sua singularidade e permitem que ele próprio se reconheça como sujeito.

A possibilidade de incluir a observação do sujeito em atividade pode aproximar o pesquisador das experiências do sujeito, pois é nela que, em geral, dá-se o confronto entre o pessoal e o coletivo. Os objetos coletivos, com seus significados e símbolos compartilhados representativos da reciprocidade entre o micro e o macro, devem ser situados na atividade concreta das pessoas, permitindo compreender o sistema singular, subjetivo, em que o sujeito reconstrói os significados de posições primeiramente sociais disponibilizados pela cultura em forma de costumes, crenças e valores. Assim ocorre com posições vivenciadas sob as marcas da exclusão e inclusão. Os mediadores semióticos utilizados para reproduzir estruturas assimétricas também têm o potencial de transformação, mas dependem da consciência e do conjunto de concepções que a pessoa posiciona nas (inter) relações.

A análise da comunicação e da metacomunicação enfatiza exatamente as instâncias da comunicação que escapam às autonarrativas. A metacomunicação relacional, entendida como comunicação sobre o próprio processo de comunicação, que informa sobre a qualidade das relações entre os parceiros da interação (Branco, Pessina, Flores,\& Salomão, 2004), é tão importante quanto a verbalização. A metacomunicação se refere aos aspectos que informam sobre o evento comunicativo (Branco, 2005). No plano metacomunicativo, que flui de forma contínua ao longo dos processos interativos, especialmente em nível não-verbal, trocamos indicadores sobre a qualidade das interações e relacionamentos que se dão em contextos culturais específicos. Quando uma professora elogia o estudante de forma indiferente e sem entusiasmo, ou então de forma irônica, certamente o resultado desse elogio será muito diverso daquele que transmite entusiasmo e admiração genuína.São exatamente esses parâmetros não verbais e para linguísticos que caracterizam a metacomunicação e que fazem toda a diferença na construção de relações entre as pessoas (Fatigante, Fasulo, \& Pontecorvo, 2004), por exemplo, relações de confiança versus desconfiança, amizade versus antipatia. Por isso, a construção das relações é muito significativa na forma como as pessoas se posicionam na experiência e como constroem suas autoteorias.

Se concebermos o self da Teoria do Self Dialógico como um sistema semiótico em uma perspectiva dinâmica, dialógica e multidimensional, assumimos que é importante estudar esse sistema na perspectiva de suas trajetórias e mudanças. Assim, consideramos inserir os estudos do self no contexto do desenvolvimento humano. Entretanto, é necessário esclarecer em qual concepção de desenvolvimento nos fundamentamos. Sabemos que nem toda mudança leva ao desenvolvimento e que a direção do desenvolvimento é multideterminada. Desenvolvimento ocorre mediante mudanças irreversíveis na organização psíquica do sujeito (Valsiner, 2001; 2007), percebidas em sua atividade, sua forma de agir e de se posicionar. Não queremos cair na armadilha de tratarmos o desenvolvimento do sistema de self dialógico de forma objetivada e como uma entidade psicológica, pois, quando nos direcionamos para um dado estado final do organismo/ sujeito, não é possível contemplar os processos envolvidos em sua emergência ou nas possibilidades de transformação. A perspectiva desenvolvimental,como elencamos a seguir, privilegia o estudo dos processos que ocorrem em tempo real ao invés de argumentos baseados em produtos de tais processos.

As teorias contemporâneas de desenvolvimento humano refletem um caráter multidimensional. A partir da segunda metade do século XX e, de forma mais consolidada, a partir do século XXI, assistimos à superação das antigas 
polarizações da teoria do desenvolvimento, construídas em torno de falsas dicotomias - natureza-criação; indivíduosociedade; biologia-cultura; cognição-afeto-típicasde uma visão determinista e reducionista. Richard Lerner (1998) destaca quatro componentes comuns às teorias atuais em desenvolvimento humano:(a) a relativa plasticidade do ser humano para mudanças sistemáticas de caráter ontogenético, ao longo da vida; (b) a multidimensionalidade da dinâmica da mudança, isto é, paraa mudança ocorrer, deve haver um rearranjo em múltiplos níveis-biológico, intrapsicológico, relacional, sociocultural, físico-ambiental etc.--; (c) a sucessão das mudanças históricas do fenômeno psicológico, em que, devido a suas combinações e fusões, cada nível configura funcionalidades específicas e diversas que assumem funções específicas para outro conjunto de fusões em outros níveis;e (d) a singularidade da experiência psicossocial, o reconhecimento de que um fenômeno psicológico percebido em um contexto específico pode não ser percebido em outro da mesma forma.

A partir dos estudos analisados, podemos perceber que a Teoria do Self Dialógico e seus desdobramentos têm contemplado muitos dos componentes preconizados pelas teorias atuais em desenvolvimento de abordagem cultural, especialmente no que se refere à multidimensionalidade e à singularidade da experiência. A transitoriedade vem sendo abordada em estudos que focam a emergência de posicionamentos novos no sistema de self a partir de mudanças concretas na vida das pessoas, como a maternidade (Duarte \& Gonçalves, 2012). Entretanto, consideramos necessários estudos de caráter ontogenético, que tenham como sujeitos crianças, adolescentes e adultos em diferentes momentos da vida, assim como estudos em uma perspectiva histórica que contemple as sucessivas mudanças na reorganização das estruturas e funções psicológicas considerando os elementos da cultura como parte integrante dos processos de significação, reelaboração subjetiva e (inter)ação.

\section{Considerações Finais}

O presente artigo apresentou a Teoria do Self Dialógico. Hubert J. Hermans, em seu esforço por elaborar uma abordagem psicoterapêutica para trabalhar com seus clientes, buscou nos trabalhos clássicos de William James, George H. Mead e Mikailanovich Bathkin conceitos e uma forma de trabalho que possibilitaram compreender o funcionamento psicológico humano do adulto em momentos de crise por meio da análise do self em uma perspectiva eminentemente dialógica. Entretanto, como em toda abordagem relacional, muitos desafios emergem, tanto de ordem teórica como de ordem metodológica. Ainda assim, a proposta tem se mostrado consistente ao organizar as relações entre o eu, o mim e o meu em um quadro de referência teórica articulada nas noções de vozes, polifonia e dialogismo. O self dialógico é estudado a partir de autonarrativas e os processos de significado são abordados mediante a identificação do sistema de valoração emergente dos discursos. Dos aspectos metodológicos, o artigo analisou o processo de construção dos RPP e a matriz de significados/valores e afetos, que são as ferramentas de análise para a identificação e caracterização dos posicionamentos pessoais assumidos pela pessoa. Todo o processo, em contexto psicoterapêutico, ao estilo rogeriano, consiste no estabelecimento de um ambiente de diálogo entre psicoterapeuta e cliente, assim comona participação ativa deste na construção, análise e reflexão do próprio sistema semiótico pessoal na esperança de auxiliar a pessoa em uma tomada de decisão.

Com um número cada vez maior de pesquisadores interessados na abordagem dialógica, a teoria tem experimentado uma consolidação e significativa expansão, incluindo a comunidade internacional fora do eixo EuropaEstados Unidos. O diálogo tem apontado para as limitações teórico-metodológicas da abordagem original no momento em que se considera o estudo do self em culturas diversas e em organizações sociais alternativas às hegemônicas sociedades dos países industrializados e institucionalizados. Porém, argumentamos que não são os aspectos conceituais, teóricos nem metodológicos, mas as concepções de mundo e de ser humano que fazem a diferença quanto às formas de construção do conhecimento psicológico.

Por isso, argumentamos a favor da expansão do self dialógico por meio da ênfase ao sistema semiótico construído nas interações e ênfase à perspectiva desenvolvimental. O desejo de participar do ambiente de comunicação é que impulsiona a tomada de determinados posicionamentos pessoais. Enquanto isso, os objetivos gerais e as práticas específicas do grupo, ao direcionarem concretamente as atividades de seus membros, retroalimentam posicionamentos por meio de ferramentas simbólicas legitimadas nas interações. O posicionamento pessoal em determinada situação deve ser visto com base em seu campo semiótico e na perspectiva desenvolvimental, pois é relativo ao processo de construção de disposições relacionais. Aceitar e rejeitar a posição que lhe é atribuída faz parte de um processo semiótico gerado e transformado na atuação das pessoas em seus contextos.

Os processos de comunicação e metacomunicação, por produzirem um contínuo fluxo de significações e ressignificações, estão relacionados à questão do desenvolvimento de um sistema de self dialógico por direcionar os significados e canalizar valores e crenças oriundos da cultura coletiva para o campo da experiência individual. Comunicação e metacomunicação estão, portanto, no centro do movimento bidirecional de feedback e feedforward de uma visão sistêmica de desenvolvimento. Além disso, é importante sublinhar que a categoria motivação passa a ocupar uma posição central na ontogênese dos processos desenvolvimentais. Por fim, cremos queo estudo do sistema de self dialógico possibilite a compreensão de categorias ou mecanismos psicológicos representativos da plasticidade e da capacidade extraordinária de mudança do ser humano.

\section{Referências}

Bakhtin, M. (1992). Marxismo e filosofia da linguagem. São Paulo: Hucitec. 
Barresi, J. (2002). From 'the thought is the thinker' to 'the voice is the speaker': William James and the dialogical self. Theory \& Psychology, 12(2), 237-250.

Bertau, M.C. (2004). Developmental origins of the dialogical self: Some significant moments. In H.J.M. Hermans \& G. Dimaggio (Eds.), The dialogical self in psychotherapy (pp. 29-42). New York: Brunner \& Routledge.

Bhatia, S.,\& Ram, A. (2001). Locating the dialogical self in the age of transnational migrations, border crossing and diasporas. Culture \& Psychology, 7(3), 297-309.

Branco, A.U. (2005). Peer interactions, language development and metacommunication. Culture \& Psychology, 11(4), 415-429.

Branco, A.U., Pessina, L., Flores, A.,\& Salomão, S. (2004). A sociocultural constructivist approach to metacommunication in child development. In A. U. Branco \& J. Valsiner (Eds.), Communication and metacommunication in human development (pp. 3-32). Greenwich.: Information Age Publishing Inc.

Chan, S.F., Ho, D.Y., Peng, S.,\& Ng, A.K. (2001). The dialogical self: Converging East-West constructions. Culture \& Psychology, 7(3), 393-408.

Chaudhary, N. (2003). Speaking the self into becoming? Culture \& Psychology, 9(4), 471-486.

Chaudhary, N.,\& Sriram, S. (2001). Dialogues of the self. Culture \& Psychology, 7(3), 379-392.

Colapietro, V. (2006). Practice, agency, \& sociality: An orthogonal reading of classical pragmatism. International Journal for Dialogical Science, 1(1), 23-31.

Duarte, F. \& Gonçalves, M. M. (2012). Negotiating motherhood: Practices and discourses. In A. C.Bastos, K. Uriko, \& J. Valsiner (Eds.), Cultural dynamics of women's lives (Book series: Advances in cultural psychology, Editor: Jaan Valsiner) (pp. 387-420). Charlotte: InformationAge Publishing.

Fatigante, M., Fasulo, A., \& Pontecorvo, C. (2004). This is not a dinner: Metacommunication in family dinnertime conversations. In A.U. Branco \& J. Valsiner (Eds.), Communication and metacommunication in human development (pp. 33-81). Greenwood: Information Age Publishers.

Gonçalves, M.,\& Salgado, J. (2001). Mapping the multiplicity of the self. Culture \& Psychology, 7(3), 367-377.

Hermans, H. J. M. (1987). Self as an organized system of valuations: Toward a dialogue with the person. Journal of Counseling Psychology, 34, 10-19.

Hermans, H.J.M. (1989). The meaning of life as an organized process. Psychotherapy, 26, 11-22.

Hermans, H.J.M. (1992). Unhappy self-esteem: A meaningful exception to the rule. Journal of Psychology, 126, 555-570.

Hermans, H.J.M. (1996a). Opposites in a dialogical self: Constructs as characters. Journal of Constructivist Psychology, 9, 1-26.

Hermans, H.J.M. (1996b). Voicing the self: From information processing to dialogical interchange. Psychological Bulletin, 119, 31-50.

Hermans, H.J.M. (2001a). The construction of a personal position repertoire: Method and practice. Culture \& Psychology, 7(3), 323-365.

Hermans, H.J.M. (2001b). The dialogical self: Towards a theory of personal and cultural positioning. Culture \& Psychology, $7(3), 243-282$.
Hermans, H.J.M. (2002). The person as a motivated storyteller: Valuation theory and the self-confrontation method. In R.A. Neimeyer \& G.J. Neimeyer (Eds.), Advances in personal construct psychology: New directions and perspectives (pp. 3-38). Westport: Praeger.

Hermans, H.J.M.,\& Dimaggio, G. (2007). Self, identity, and globalization in times of uncertainty: A dialogical analysis. Review of General Psychology, 11, 31-61.

Hermans, H.J.M.,\& Dimaggio, G. (Eds.) (2004). The dialogical self in psychotherapy. New York: Brunner \& Routledge.

Hermans, H.J.M.,\& Hermans-Jansen, E. (1995). Self-narratives: The construction of meaning in psychotherapy. New York: Guilford Press.

Hermans, H.J.M.,\& Kempen, H.J.G. (1993). The dialogical self: Meaning as movement. San Diego: Academic Press.

Hermans, H.J.M., Kempen. H.J.G., \& Van Loon, R. (1992). The dialogical self: Beyond individualism and rationalism. American Psychologist, 47, 23-33.

Hermans, H. J. M., Rijks, T., \& Kempen, H. J. G. (1993). Imaginal dialogues in the self: Theory and method. Journal of Personality, 61, 207-235.

Holquist, M. (1990). Dialogism: Bakhtin and his world. London: Routledge.

James, W. (1890/2007). The consciousness of self. In The principles of psychology (pp. 291-401). New York: Cosimo.

Lerner, R. (1998). Theories of Human Development: Contemporary Perspectives. In W. Damon \& R. Lerner (Eds.), Handbook of child psychology: Theoretical models of human development ( $5^{\text {a }}$ ed., pp. 1-24). New York: John Wiley \& Sons, Inc.

Lima de Souza, M. \& Gomes, W. B. (2009). Temporalidade e espacialidade na estrutura do self nas abordagens semiótica e dialógica. Psicologia em Estudo, 14(2), 365-373.

Mead, G. H. (1913). The social self. Journal of Philosophy, 10, 374-380.

Mead, G.H. (1967). Mind, self, and society. Chicago: University of Chicago Press.(Trabalho original publicado em 1934)

Osatuke, K., Gray, M, Glick, M., Stiles, W.B., \& Barkham, M. (2004). Hearing voices: Methodological issues in measuring internal multiplicity. In H. J. M. Hermans \& G. Dimaggio (Eds.), The dialogical self in psychotherapy (pp. 237-254). New York: Brunner \& Routledge.

Salgado, J. (2006). Listening to India, listening to ourselves: The place of self in culture. Culture \& Psychology, 12(1), 101-113.

Valsiner, J. (2001). Contemplating self: From India to contemporary self-psychology. Culture \& Psychology, 7(1), 115-118.

Valsiner, J. (2007). Culture in minds and societies: Foundations of Cultural Psychology. New Delhi: Sage.

Recebido em 13.03.2014

Primeira decisão editorial em 31.05.2015

Aceito em 31.05.2015 